\title{
Helicobacter pylori seropositivity in subjects with acute myocardial infarction
}

\author{
Barrie Rathbone, Daniel Martin, Jonathan Stephens, John R Thompson, Nilesh J Samani
}

\begin{abstract}
Objective-To determine whether Helicobacter pylori infection increases the risk of myocardial infarction.

Design-Case-control study.

Setting-University teaching hospital. Methods-Serological evidence of $H$ pylori infection was determined in 342 consecutive patients with acute myocardial infarction admitted into the coronary care unit and in 236 population-based controls recruited from visitors to patients on medical and surgical wards. Results-206/342 (60.2\%) of cases were $H$ pylori positive compared with 132/236 $(55.9 \%)$ of controls $(P=0 \cdot 30)$. Age and sex stratified odds ratio for myocardial infarction associated with $H$ pylori seropositivity was 1.05 (95\% CI 0.7 to 1.53 , $P=0.87)$ and this remained non-significant $(P=0.46)$ when other risk factors for ischaemic heart disease were taken into account using logistic regression analysis. $H$ pylori seropositivity was not associated with several coronary risk factors in either cases or controls.

Conclusion-No increase was found in $H$ pylori seropositivity in subjects with acute myocardial infarction. This suggests that previous $H$ pylori infection is not a major risk factor for acute myocardial infarction.
\end{abstract}

(Heart 1996;76:308-311)

Keywords: Helicobacter pylori infection; myocardial infarction; cardiovascular risk factors

Among the more intriguing factors to have been linked to the risk of coronary heart disease (CHD) are chronic bacterial infections, ${ }^{12}$ and recently it has been suggested that Helicobacter pylori colonisation from childhood may relate to CHD risk. ${ }^{3} H$ pylori is now recognised as the major cause of histological chronic gastritis and a necessary factor in the development of duodenal and gastric ulceration. Successful eradication of $H$ pylori makes ulcer relapse extremely unlikely.

Until recently, the data supporting a relation between $H$ pylori and CHD relied on relatively small case-control studies, ${ }^{3-5}$ although two larger conflicting studies ${ }^{67}$ have just been reported (see Discussion). To investigate further the association between $H$ pylori colonisation and CHD we have compared 342 patients with acute myocardial infarction (MI) with
236 control subjects for evidence of previous $H$ pylori infection.

\section{Patients and methods} SUBJECTS

Patients who satisfied the World Health Organisation criteria for $\mathrm{MI}$ in terms of symptoms, enzyme increases, or electrocardiographic changes ${ }^{8}$ were recruited from admissions to the coronary care unit (CCU) at Leicester Royal Infirmary. The CCU, serving a population of around 300000 , accounts for $>65 \%$ of admissions of cases with $\mathrm{MI}$ in Leicester. The period of recruitment was between July 1993 and April 1994, and > 97\% of eligible subjects were recruited.

Control subjects were recruited from adult visitors to patients with non-cardiovascular illnesses on general medical and surgical wards at the Leicester Royal Infirmary to provide subjects likely to be representative of the source population from which the cases came. A note was made of controls who reported a history of coronary heart disease but such subjects were not excluded. Cases and controls filled in a standard questionnaire about their personal histories, had height and weights measured, and provided blood samples for measurement of serum total and HDL cholesterol and $H$ pylori IgG antibodies.

The study was approved by the local clinical research ethics committee.

BIOCHEMICAL MEASUREMENTS

Serum total and HDL-cholesterol were measured using a Kodak Ektachem E700 CXR Automatic Analyser in a quality-controlled hospital biochemistry laboratory. For cases, the first blood sample taken after admission was used for the analysis.

\section{DETERMINATION OF H PYLORI STATUS}

$H$ pylori status was established serologically using an established IgG ELISA assay based on an ultracentrifuged sonicate antigen with a sensitivity of $92.3 \%$ and a specificity of $90 \cdot 7 \% .{ }^{9}$ The sensitivity and specificity values were established using a local Leicester-based dyspepsia population with $H$ pylori status being determined by a combination of histology, culture, and urease testing. All samples were assayed in duplicate and samples with borderline values were assessed further by immunoblotting. 
Table 1 Seropositivity for Helicobacter pylori infection in acute myocardial infarction cases and population controls

\begin{tabular}{llc}
\hline Group & Cases & Controls \\
\hline Males & $135 / 229(59 \cdot 0 \%)$ & $81 / 141(57 \cdot 4 \%)$ \\
Females & $71 / 113(62 \cdot 8 \%)$ & $51 / 95(53 \cdot 7 \%)$ \\
Total & $206 / 342(60 \cdot 2 \%)$ & $132 / 236(55 \cdot 9 \%)$ \\
\hline
\end{tabular}

qualitative risk factors between cases and controls or qualitative risk factors between $H$ pylori positive and $H$ pylori negative cases or controls were compared using the chi-squared test. Quantitative sample means were compared by analysis of variance. Odds ratios and $95 \%$ confidence intervals (CI) estimating the relative risk of $\mathrm{MI}$ associated with $H$ pylori seropositivity were calculated using the Mantel-Haenszel method with stratification for age and sex. Logistic regression was used to analyse the influence of other factors on the odds ratio.

\section{Results}

$206 / 342(60 \cdot 2 \%)$ of patients and $132 / 236$ (55.9\%) of controls were $H$ pylori seropositive (odds ratio $1.19(95 \%$ CI 0.84 to 1.69$) \mathrm{P}=$ 0.30 ) (table 1 ). Controls were significantly younger than patients $(56.0(13.0)(\mathrm{SD})$ years versus $65.1(11.7)$ years, $P<0.001)$. As reported previously, ${ }^{7}$ seropositivity increased with age in both our groups (data not shown), making the age-stratified odds ratio even less significant (odds ratio 1.05 (95\% CI 0.72 to 1.53), $P=0.87$ ).

Table 2 shows the demographic and coronary risk factors in cases and controls grouped by $H$ pylori serological status. All the classic risk factors for CHD were significantly increased $(P<0.01)$ in cases compared with controls. However, there was no difference in either cases or controls when the subjects were classified according to $H$ pylori status (table 2 ). In logistic regression analysis the association of $H$ pylori positivity with myocardial infarction remained non-significant when adjusted for these risk factors (odds ratio $0.85,95 \%$ CI 0.55 to $1.31, P=0.46)$.

Leicester has a considerable ethnic community: $11.9 \%$ of cases and $6.3 \%$ of controls were non-white. As a whole, $H$ pylori seropositivity was higher in non-white subjects $(67 \cdot 8 \%)$ than in white subjects $(57.5 \%)$ although this did not reach statistical significance $(P=0 \cdot 13)$. In each group, however, there was no difference in frequency of $H$ pylori infection between cases and controls $(59.1 \% v 55.2 \%$ for white cases and controls respectively and $68.3 \% v 66.2 \%$ for non-white cases and controls). Thus the age and sex stratified odds ratio for white subjects only was 1.05 (95\% CI, 0.71 to $1.54, P=0.88$ ). Similarly, the association remained non-significant when only young $(<55$ years, median age for controls) cases and controls were compared (odds ratio $1 \cdot 26,95 \% \mathrm{CI} 0.66$ to $2 \cdot 41, \mathrm{P}=0.45$ ).

\section{Discussion}

There are considerable problems in investigating potential associations between $H$ pylori and CHD. ${ }^{10}$ Both conditions are common in the population, both increase with age, and both are associated with socioeconomic status. A causal association between $H$ pylori and CHD if proven is, however, very important in that $H$ pylori is easily screened for and amenable to treatment. There is also the potential for a future vaccine which could be used in childhood, because it is thought that $H$ pylori is predominantly transmitted in childhood. ${ }^{11}$

Previous studies ${ }^{3-7}$ examining the relation between $H$ pylori and $C H D$ have used a variety of criteria to define the presence of $\mathrm{CHD}$. Thus in the original report demonstrating an association, subjects with angiographically defined CHD were studied. ${ }^{3}$ More recently, in a cross-sectional study of a population based sample of 388 men, the same group found an association when CHD was defined on the basis of either electrocardiographic or symptomatic/historical criteria. ${ }^{6}$ Interestingly, the association was strongest when CHD was defined on the basis of ECG changes which included any of the following: $Q$ waves, $S T$ segment depression, left bundle branch block, or $\mathrm{T}$ wave inversion. The association with ECG changes remained significant when adjusted for other factors, whereas that with history of angina/MI became non-significant after adjustment. Since several of the ECG

Table 2 Comparison of demographic and coronary risk factors in cases and controls grouped by Helicobacter pylori serological status

\begin{tabular}{|c|c|c|c|c|c|c|}
\hline & \multicolumn{3}{|l|}{ Cases } & \multicolumn{3}{|l|}{ Controls } \\
\hline & HP+ve & $H P-v e$ & $P$ & HP+ve & $H P-v e$ & $P$ \\
\hline $\begin{array}{l}\text { Age } \\
\text { Males }(\%) \\
\text { White }(\%) \\
\text { Current smokers }(\%)^{\star} \\
\text { Angina }(\%)^{\star} \\
\text { Hypertension }(\%)^{\star} \\
\text { Diabetes }(\%)^{\star} \\
\text { +ve FH }(\%)^{\star} \\
\text { BMI } \dagger \\
\text { Total cholesterol } \dagger \\
\text { HDL cholesterol } \dagger\end{array}$ & $\begin{array}{l}65 \cdot 2(11 \cdot 5) \\
65 \cdot 5 \\
86 \cdot 4 \\
31 \cdot 7 \\
28 \cdot 9 \\
33 \cdot 9 \\
12 \cdot 2 \\
36 \cdot 7 \\
26 \cdot 2(4 \cdot 2) \\
5 \cdot 5(1 \cdot 3) \\
1 \cdot 20(0 \cdot 36)\end{array}$ & $\begin{array}{l}64 \cdot 8(12 \cdot 1) \\
69 \cdot 1 \\
90 \cdot 4 \\
33 \cdot 6 \\
27 \cdot 6 \\
32 \cdot 0 \\
11 \cdot 6 \\
41 \cdot 6 \\
24 \cdot 9(4 \cdot 1) \\
5 \cdot 6(1 \cdot 1) \\
1 \cdot 25(0 \cdot 34)\end{array}$ & $\begin{array}{l}0.76 \\
0.49 \\
0.26 \\
0.73 \\
0.83 \\
0.73 \\
0.84 \\
0.45 \\
0.02 \\
0.57 \\
0.31\end{array}$ & $\begin{array}{l}57 \cdot 4(13 \cdot 1) \\
61 \cdot 4 \\
92 \cdot 4 \\
20 \cdot 6 \\
4 \cdot 9 \\
19 \cdot 1 \\
6 \cdot 9 \\
26 \cdot 7 \\
25 \cdot 7(3 \cdot 6) \\
5 \cdot 7(1 \cdot 1) \\
1 \cdot 16(0 \cdot 29)\end{array}$ & $\begin{array}{l}54 \cdot 4(12 \cdot 5) \\
57 \cdot 7 \\
95 \cdot 2 \\
14 \cdot 4 \\
9 \cdot 2 \\
16 \cdot 3 \\
1.0 \\
22 \cdot 7 \\
25 \cdot 1(3 \cdot 6) \\
5 \cdot 6(1 \cdot 1) \\
1 \cdot 23(0 \cdot 37)\end{array}$ & $\begin{array}{l}0.08 \\
0.57 \\
0.39 \\
0.22 \\
0.22 \\
0.59 \\
0.03 \\
0.44 \\
0.31 \\
0.61 \\
0.15\end{array}$ \\
\hline
\end{tabular}

HP+ve, $H$ pylori seropositive; $\mathrm{HP}-\mathrm{ve}, H$ pylori seronegative; $\mathrm{FH}$, family history of $\mathrm{MI}$ in first degree relative; $\mathrm{BMI}$, body mass index $\left(\mathrm{kg} / \mathrm{m}^{2}\right)$. 
changes included could have non-CHD causes, this raises questions about the specificity of the observed association.

In the largest study to date, Murray et al recently reported their findings in participants recruited in Northern Ireland as part of the MONICA project. ${ }^{7}$ In 2115 subjects between the ages of 25 and $64, H$ pylori seropositivity was $57 \cdot 6 \%$. 135 subjects $(6 \cdot 4 \%)$ had $\mathrm{CHD}$ according to the Rose angina questionnaire. After adjustment for age, sex, social class, smoking, and HDL-cholesterol, the odds ratio for CHD for subjects who were $H$ pylori seropositive was 1.51 (95\% CI, 0.93 to 2.45 , P $=0 \cdot 1)$. The authors concluded that they could not entirely exclude an important association between $H$ pylori infection and CHD but that if one did exist it could not account for the social class inequality in CHD.

In the current study only cases who strictly satisfied the WHO criteria for acute MI were recruited. Subjects were recruited prospectively with $>97 \%$ recruitment of eligible subjects to provide the largest number of cases so far in whom an association with $H$ pylori seropositivity has been examined. Within the confidence intervals of the odds ratios we did not find a significant association between $H$ pylori infection and risk of acute MI in either the whole cohort or selected subgroups.

A negative study always raises questions about the power of the study. A priori our study had $80 \%$ power at a $\mathrm{P}$ value of 0.05 to detect a $1 \cdot 6$-fold increase in risk. As indicated by the $95 \%$ confidence intervals obtained for the odds ratios, our study cannot exclude an effect of a lower magnitude of previous $H$ pylori infection on $\mathrm{MI}$ risk.

A critical feature of any case-control study is the selection of control subjects. They need to provide a reliable estimate of the prevalence of a putative risk factor in the base population from which the cases are recruited. We therefore chose to recruit our controls from individuals visiting patients in the same hospital. Controls were recruited concurrently with the myocardial infarction cases. Using information on the age and sex distribution of myocardial infarction cases admitted to the CCU in the previous year held in our CCU database, we attempted to recruit controls of a mix similar to that of the likely myocardial infarction cases, although no individual willing to participate was excluded. The prevalence of $H$ pylor $i$ seropositivity in our controls $(55.9 \%)$ is similar to that observed by Murray et $a l^{7}$ in their cross-sectional study.

Adjustment for several risk factors for CHD did not alter the odds ratio for risk of $\mathrm{MI}$ associated with $H$ pylori seropositivity. However, a potentially important limitation of our study is lack of information on social class of cases and controls. Prevalence of $H$ pylori seropositivity increases from social class I to $V .{ }^{710}$ Thus despite our efforts to recruit suitable controls (see above), if the cases and controls differed significantly in this variable, it could have masked an association. We cannot exclude this possibility, although it is interesting to note that in their study, Murray et $a l^{7}$ found that after age and sex were adjusted for, social class did not markedly affect their odds ratio for the association of $H$ pylori seropositivity with CHD.

Another possible explanation for our negative finding could be our choice of cases. As mentioned earlier, some previous studies have included not only subjects with $\mathrm{MI}$, but also cases with CHD diagnosed by other criteria. It is possible that $H$ pylori infection predisposes primarily to atheroma formation and the association with the downstream phenotype of $\mathrm{MI}$, which has other important contributing factors such as thrombosis, is much weaker. However, against this, we found no increase in prevalence of $H$ pylori seropositivity in cases with a previous history of angina (table 2) and the odds ratio with $\mathrm{MI}$ remained non-significant when the analysis was restricted to these subjects (data not shown).

Several conventional risk factors were, as expected, more prevalent in our cases compared with controls. However, as reported previously, ${ }^{67}$ we found no association between $H$ pylori serological status and these factors. To explain the relation between $H$ pylori and CHD, most attention has focused on acute phase reactants such as fibrinogen, which may be increased in such chronic infections and which in turn may predispose to CHD. Thus, in two separate studies, Patel et $a l^{612}$ found higher circulating fibrinogen concentrations in $H$ pylori seropositive individuals compared with $H$ pylori negative subjects. However, in their much larger cohort, Murray et $a l^{7}$ found no such increase, and, if anything, there was a weak negative association. Therefore, in addition to controversy regarding the existence of a relation between $H$ pylori and CHD there is also conflicting data regarding the effect of $H$ pylori infection on the favourite intermediary phenotype.

In summary, in a large series of consecutively recruited and unselected acute myocardial infarction cases we were unable to demonstrate any association between $H$ pylori colonisation and $\mathrm{MI}$ beyond that expected in two conditions which occur commonly in the population and increase with age. This raises serious doubt regarding the association with $\mathrm{CHD}$, and on current evidence one cannot use risk of CHD to justify widespread $H$ pylori eradication.

1 Mattila KJ, Nieminen MS, Valtonen VV, Rasi VP, Kesaniemi YA, Syrijala SL, et al. Association between dental health and myocardial infarction. BMF 1989;
298:779-82. 298:779-82.

Saikku P, Leinonen M, Mattila K, Ekman M-R, Nieminen MS, Makela PH, et al. Serological evidence of an association of a novel chlamydia, TWAR, with chronic coronary heart disease and acute myocardial infarction. Lancet 1988;ii:983-6.

3 Mendall MA, Goggin PM, Molineaux N, Levy J, Toosy T, Strachan D, et al. Relation of Helicobacter pylori infection and coronary heart disease. Br Heart f 1994;71:437-9.

4 Morgando A, Sanseverino P, Perotto C, Molino F, Gai V, Ponzetto A. Helicobacter pylori seropositivity in myocardial infarction. Lancet 1995;345:1380.

5 Martin-de-Argila C, Boixeda D, Canton R, Gisbert JP, Fuertes A. High seroprevalence of Helicobacter pylor infection in coronary heart disease. Lancet 1995;346:310.

6 Patel P, Mendall MA, Carrington D, Strachan JP, Leatham E, Molineaux N, et al. Association of Helicobacter pylor and Chlamydia pneumoniae infections with coronary heart disease and cardiovascular risk factors. $B M F 1995 ; 311$ $711-4$. 
7 Murray LJ, Bamford KB, O'Reilly DPJ, McCrum EE, Evans AE. Helicobacter pylori infection: relation with cardiovascular risk factors, ischaemic heart disease, and social class. Br Heart f 1995;74:497-501.

8 WHO (Report of the joint International Society and Federation of Cardiology/World Health Organisation Task Force on standardization of clinical nomenclature) Task Force on standardization of clinical nomenclature). heart disease. Circulation 1979;59:607-9.

9 Hirschl AM, Rathbone B, Wyatt JI, Berger J, Rotter ML. Comparison of EIA antigen preparations alone or in combination for serodiagnosing Helicobacter pylori infections. f Clin Pathol 1990;43:511-3.

10 Glynn JR. Helicobacter and the heart. Lancet 1994;344:146. 11 Mendell MA, Coggin PM, Molineaux N, Levy J, Toosy T, Strachan D, et al. Childhood living conditions and Helicobacter pylori seropositivity in adult life. Lancet 1992;339:896-7.

12 Patel P, Carrington D, Strachan DP, Leatham E, Groggin P, Northfield TC, et al. Fibrinogen: a link between chronic infection and coronary heart disease. Lancet 1994;343: 1634-5.

\section{STAMPS IN CARDIOLOGY}

\section{Jan Evangelista Purkinje (1787-1869)}

Czechoslovakia issued two stamps in 1937 to commemorate the 150th anniversary of the birth of Purkinje (spelt Purkyne in Czech). The label (A) illustrates part of a medical design and features the staff and serpent. It is not incorporated in the stamp (B) and is frequently lost when the stamp is in used condition, the label having been torn off.

Jan Evangelista Purkinje was born in Libochovice, Bohemia, and graduated in medicine from Prague. While he was professor of physiology at the University of Breslau he made several widely acclaimed scientific discoveries. He was one of the very first to use the microscope to explore the function of tissues, "histophysiology" as he called it, and he introduced the term protoplasm. In 1839 he described the subendocardial structures in the heart, known ever since as the Purkinje fibres. Later it was realised that the network of Purkinje can be seen with the naked eye in the sheep and the ox, and the whole arborisation shown clearly by injecting the sheaths of the fibres with India ink. S Tawara identified the atrioventricular node in 1906 and showed that the AV bundle was prolonged into the Purkinje network. A few years later it was possible with Einthoven's invention of the electrocardiograph to quantify the rate of conduction and rhythmicity in the Purkinje substance. Purkinje has ten other eponymous descriptions to his name, the best known being the cells in the cerebellum. He described the classification of fingerprints and their importance for identification.

M K DAVIES

A HOLLMAN
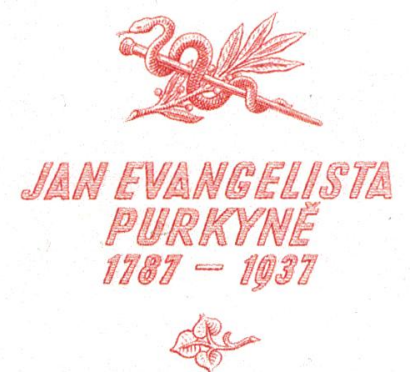

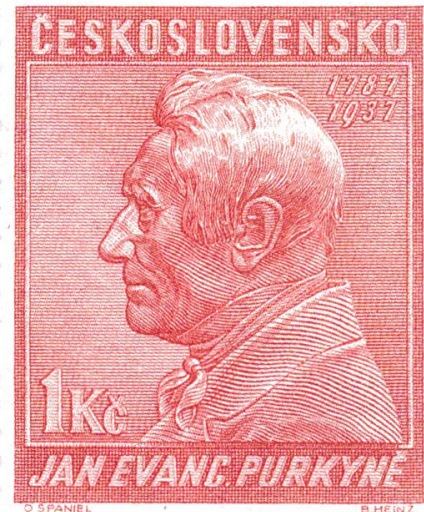

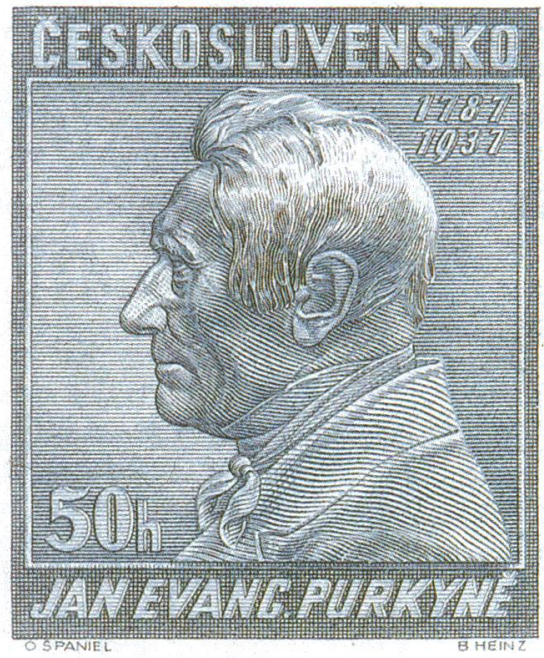

